\title{
Sequential effects in absolute judgments of loudness'
}

MORRIS K. HOLLAND AND G. R. LOCKHEAD

DUKE UNIVERSITY
The effects of preceding stimuli on the judgments of current stimuli were examined in a sludy using absolute judgments of loudness with feedback. It was found that the response on a given trial was dependent on the stimuli in the preceding sequence of at least five trials. Both assimilation and contrast effects were observed. The form of the dependency of a response on a prior stimulus was a function of the ordinal position of the stimulus in the preceding sequence of trials. The stimulus on the immediately preceding trial had an assimilative effect on the response and preceding stimuli two to five trials removed all showed a contrast effect on a given response. The extent to which these preceding stimuli contributed to the contrast effect was an increasing function of their recency. The reversal of the dependency of the response, from assimilation to the stimulus one trial back, to contrast with the stimuli two and more trials back, indicates a unique function of the immediately preceding stimulus in this task. Since there was a reduction in the variance of responses to those stimuli similar in value to the immediately preceding stimulus, it is proposed that the stimulus and feedback on the last trial were remembered and used as a standard in judging the presented stimulus. A model is presented in which it is assumed that the memory of the magnitude of the immediately preceding stimulus is contaminated in specified ways by prior stimuli in the series. The empirical findings of assimilation and contrast are expected consequences of the proposed memorial processes.

The dependency of the response on the preceding sequence of stimuli has been examined in several psychophysical tasks. A consistent inverse relation, contrast, has been reported between responses and the average value of all preceding stimuli when feedback is not provided (Helson, 1959). With no feedback, contrast also occurs between the response and the immediately preceding stimulus (Pollack, 1964; Parducci \& Sandusky, 1965). When feedback is given, however, a direct relation, or assimilation, is typically found between the response and the immediately preceding stimulus. Parducci and Marshall (1962) and Barry (1964) investigated the absolute judgments of weights, with information feedback, and reported assimilation; i.e., responses varied directly with the value of the preceding stimulus. Garner (1953), for absolute judgments of loudness, showed a similar assimilative effect with Ss who received no feedback but who had previously been highly trained under feedback conditions.
Parducci and Marshall (1962) show that these two effects are consistent with adaptation-level theory if it is assumed that, when response usage is highiy specified in an absolute judgment task (e.g., with feedback), Ss treat the stimulus one trial back as they treat a standard in a comparative judgment task. If this assumption is correct, both types of sequential effects may also be explained by a consideration of only memorial processes. For an absolute judgment task with feedback, the problem for the Ss may be to retrieve the value of the stimulus one trial back (the standard) from an imperfect memory. Sequential effects would be observed if the memory of the standard were affected by traces from the memories of previous stimuli. It will be shown that with this type of faulty memory, both assimilation and contrast are expectable in the same sequence of trials if feedback is provided; that is, responses should vary directly with the values of the stimuli which immediately precede them (assimilation), and should vary inversely with preceding stimuli two or more trials removed (contrast). The data reported below show this dual effect.

\section{METHOD}

The task was one of absolute judgments. Ten loudness stimuli were identified with the numbers 1 through 10 , with the quietest value labeled number 1 and the loudest labeled number 10. Each $S$ initiated presentation of the randomly selected stimulus with a foot switch, recorded his response on a 10-key typewriter which he could operate by touch, and then recorded the correct response which appeared, $100 \mathrm{msec}$ after the response was recorded, as an illuminated numeral in a tachistoscope. Keying the knowledge of results turned that light off immediately. A buzzer sounded for $0.75 \mathrm{sec}$ after the stimulus value was recorded. The Ss could present the next stimulus any time after the end of the buzzer and generally did so immediately.

The stimuli were ten $0.5 \mathrm{sec} 1200 \mathrm{cps}$ sinusoids generated by a Hewlett-Packard $200 \mathrm{CD}$ oscillator and varied in intensity by attenuators with impedance matching transformers. The stimuli were presented through high quality low impedance earphones. The total range of the 10 loudnesses used was measured as $25 \mathrm{~dB}$ re $0.0002 \mathrm{dyne} / \mathrm{cm}^{2}$. The separate loudnesses are not given because available equipment did not provide precise determination. The loudnesses used were judged by $S s$ in earlier pilot work 
to be of 10 subjectively equal steps, and the data do not suggest any marked departure from this equality. Discrimination was difficult and responses were correct about one-third of the time.

Important for the analyses reported here is the success obtained in random presentation of the stimuli. The selection of stimuli was accomplished by stopping a $75 \mathrm{kc}$ clock which cascaded a pulse, one position per cycle, through a 10-position diode matrix. The number selected was determined by the position of the pulse when the clock was stopped, one position for each loudness. To eliminate the chance that $\mathrm{Ss}^{\prime}$ responding could become so rhythmic as to stop the clock at the same position, the circuitry was additionally modulated by a Zener-type noise diode. Separate analyses of the stimulus sequence revealed no suggestion of nonrandomness.

The Ss were three male psychology graduate students who had had previous experience in absolute judgment tasks and were paid for participating. Each of the three Ss made 150 loudness judgments in each of 18 sessions for a total of 2700 reponses per S, 8100 responses for all three Ss.

The data reported here were collected from three conditions of two successive experiments. Each experimental session lasted about $55 \mathrm{~min}$, but is performed in the conditions reported here only during one-third of each session. The position of the reported condition in the sessions was balanced over Ss and days. Because other conditions in the studies involved visual discriminations, all Ss looked into a tachistoscope with a blank field during the reported conditions. The field was lighted for two of the conditions and dark for the third. Since there was no indication that the differences in field illumination affected performance on the loudness judgments, the data were collapsed over these conditions to provide a substantial number of observations. All effects reported here are demonstrable, ilthough more variable, with each of the separate sets of data.

\section{Assimilation}

\section{RESULTS}

The results indicate that assimilation occurred between the value of the preceding stimulus and the response to the presented stimulus. Figure 1 shows the average systematic error of response as a function of the stimulus on the immediately preceding trial. The average response to each stimulus was drawn toward the value of the preceding stimulus and this effect was greatest for stimuli in the middle of the series. For example, the average response to stimuli 5 and 6 was 5.5 (zero average error) following the occurrence of stimulus 5 or 6 ; however, the average response to these stimuli was 6.3 (plus .8 average error) following stimulus 9 or 10 , and 4.7 (minus .8 average error) following stimulus 1 or 2. Each point in

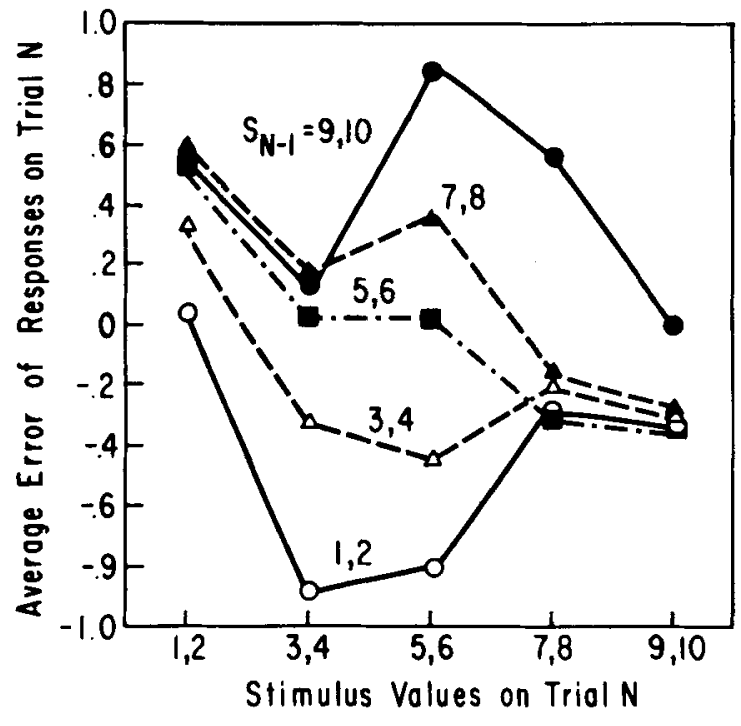

Fig. 1. The average systematic error of responses on trial $\mathbf{N}$ as a function of the value of the stimulus on trial N-1. The data are collapsed over pairs of stimuli.

Fig. 1 represents approximately 300 observations summed over the three Ss. The data were collapsed over pairs of values to decrease variability in the figures.

First-order sequential dependencies were examined in more detail to determine the adequacy of certain possible explanations of the observed assimilative effect. One possible explanation is that the $S$ may overcorrect in shifting his response scale following an error. For example, an error in response to stimulus 1 must be at high error since no response of zero was allowed; if the $S$ overcorrected on the following trial, he would tend to make a low error. The tendency to make low errors following a low stimulus can be seen in Fig. 1 and is the assimilative effect observed. To test the overcorrection hypothesis, responses following low errors were compared with responses following high errors. No significant difference was observed. For example, when responses were two steps too high, the average response on the following trial was 5.49; when responses were two steps too low, the average response on the following trial was 5.46. High and low errors were essentially equal for both cases. Thus the direction of error of the response on the preceding trial apparently does not determine the sequential effect. The average expected response was 5.5 , which is not reliably different than either of these observations.

A second possible explanation of assimilation, suggested by Garner (1953), is that when $S$ s are uncertain of the value of the presented stimulus they may repeat their last response. In the present study, repetition both of the last response and of the last feedback was significantly above chance 


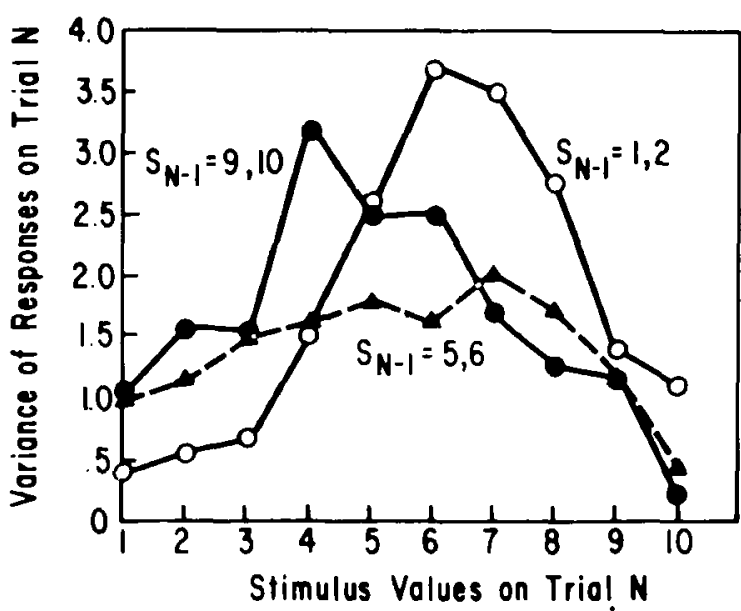

Fig. 2. Variance of responses to each stimulus value on trial $\boldsymbol{N}$ as infuenced by low, medium, or high stimulus values on trial N-1.

(response repetition was $13.8 \%, \mathrm{p}<0.001$, and feedback repetition was $14.5 \%, p<0.001$ ). While repetition occurred, more than repetition was involved in the observed assimilative effect, since there was an apparent shift of the entire response scale as a function of the preceding stimulus. The modal responses to stimuli $1-10$ on trials following the occurrence of stimulus 1 were $1,1,2,2,4,4,8$, $9,9,10$; following stimulus 10 they were $1,2,4,4$, $7,7,8,9,10,10$. Such an effect could not be due solely to the relative frequency of responses 1 and 10.

Another posstbility suggested (Garner, 1953) is that the perceived value of the presented stimulus may be some weighted mean of the physical values of the presented stimulus and the preceding stimulus. The data of the present experiment yield evidence that the involvement of the preceding stimulus in the production of the response is more than a simple averaging model would predict. Subjects apparently were able to remember something of the value of the preceding stimulus and to use that information in responding to the presented stimulus. Figure 2 shows that response variance was systematically lower when the presented stimulus was in the vicinity of the preceding stimulus than when considerably removed from it. Thus, when the preceding stimulus $\left(\mathrm{S}_{\mathrm{N}-1}\right)$ was 1 or 2 , Ss were less variable in responding to stimuli 1-4, and more variable in responding to stimuli 6-10. Aside from the attenuation in variance resulting at the ends of the scale, Fig. 2 indicates that variance was an increasing function of the absolute difference, in category steps, between the preceding stimulus $\left(\mathrm{S}_{\mathrm{N}-1}\right)$ and the presented stimulus $\left(\mathrm{S}_{N}\right)$. This result suggests that the preceding stimulus provided for a local increase in information by serving as a standard for judgment.

\section{Contrast}

The effect of a stimulus on one trial on the responses on the following trials is shown in Fig. 3. For this analysis, average responses were examined for each of eight trials lollowing those trials on which a particular stimulus occurred. Each point in Fig. 3 represents $1200-1600$ observations collapsed over the three $S s$ and shows the mean response on trial $\mathrm{N}+\mathrm{K}$ as a function of the stimulus on trial $\mathrm{N}$. Since the stimulus sequence was random, the mean stimulus on trial $N+K$ was not dependent on the stimulus on trial $\mathrm{N}$, and was approximately 5.5 for all sequences examined. Average error of responses, however, varied systematically with preceding stimuli. Thus, when stimulus 9 or 10 occurred on trial $\mathrm{N}$, the average response on trial $\mathrm{N}+1$ was .40 above the average stimulus on trial $N+1$ (assimilation) and the average response on trial $N+2$ was .20 below the average stimulus on trial $\mathrm{N}+2$ (contrast). It can be seen that the stimulus on trial $N$ had an assimilative effect on the response on trial $\mathrm{N}+1$ and a contrast effect on the response on trial $N+2$. Further, this contrast effect partially continues through trial $N+8$, which is as far as the analysis was conducted. The effect of contrast is consistent with adaptation-level theory (Helson, 1959). A recency effect can also be seen in Fig. 3. Contrast of responses with stimuli two or three trials back was stronger than with stimuli four or five trials back.

Figure 3 shows the average effects of stimuli in the preceding sequence of trials on current responses. Figure 4 shows that these average effects interact. For this figure, each response at trial $N$ was classified on the basis of the stimulus on trial $\mathrm{N}-1$ and of a weighted mean of stimuli occurring on trials $\mathrm{N}-2, \mathrm{~N}-3$, and $\mathrm{N}-4$. In calculating this mean, the stimuli were weighted for recency, with arbitrary weights of four for $\mathrm{S}_{\mathrm{N}-2}$, two for $\mathrm{S}_{\mathrm{N}-3}$, and one for $\mathrm{S}_{\mathrm{N}-4}$. The weighted mean of these stimuli was regarded for this analysis as defining a stimulus context for the judgment at trial $N$. The

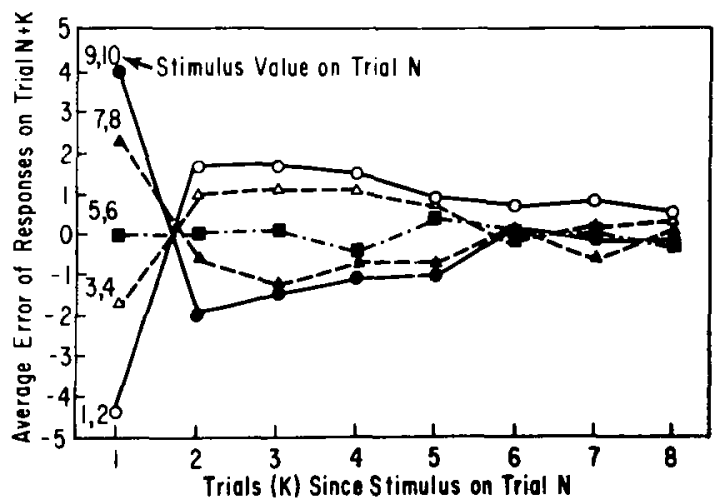

Fig. 3. The effect of the stimulus on a given trial on the responses on the next eight trials. 


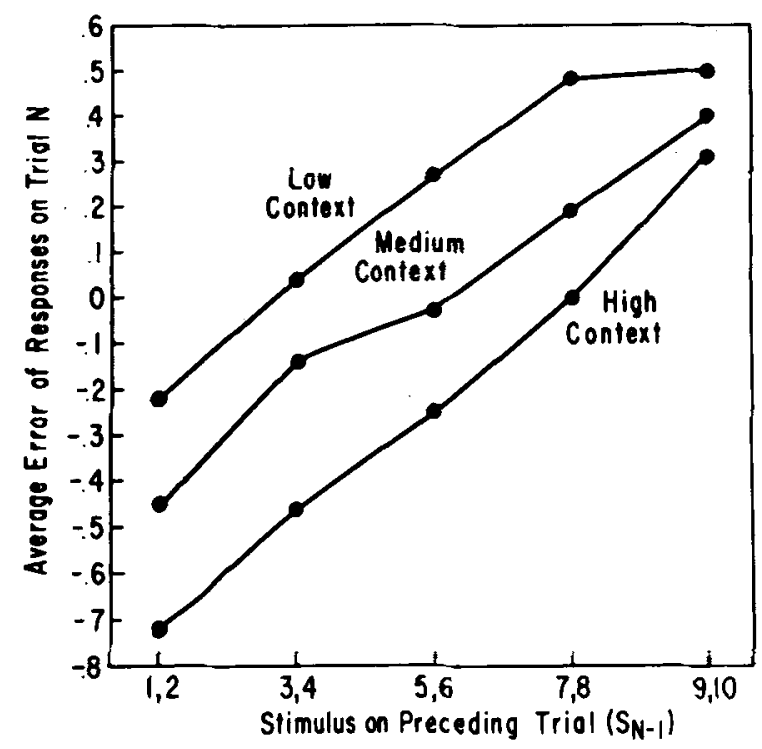

Fig. 4. Average error of response on trial $\mathbf{N}$ as a combined function of the value of the stimulus on trial $N-1$ and the preceding stimulus context as described in the text.

observations were divided into three classes: low context-average value of preceding stimuli of 1.0 to 4.4 ; medium context-average of 4.5 to 6.5 ; and high context-average of 6.6 to 10.0 . Figure 4 shows the average response at trial $\mathrm{N}$ as a function of the stimulus on trial $\mathrm{N}-1$ and the prior stimulus context (the stimuli on trials $N-2, N-3$, and $N-4$ ). The extent of the dependency of the response on prior stimulus context is revealed by the clear separation of the three functions in Fig. 4. That the relation was one of contrast can be seen from the fact that the highest prior context resulted in the lowest average response, Assimilation of the response at trial $\mathrm{N}$ and the stimulus on trial $\mathrm{N}-1$ is also apparent in Fig. 4. For each of the three functions, the average error of response on trial $N$ varied directly with the value of the stimulus on trial $\mathrm{N}-1$.

Flgures 2, 3, and 4 suggest that prior sequences of four stimuli could be selected which would result in a severe underestimation, a severe overestimation, or a marked increase or decrease in accuracy on the trials which follow them. For the sequence $\mathrm{S}_{\mathrm{N}-4}, \mathrm{~S}_{\mathrm{N}-3}, \mathrm{~S}_{\mathrm{N}-2}, \mathrm{~S}_{\mathrm{N}-1}, \mathrm{~S}_{\mathrm{N}}$, a weighted average of $S_{N-4}, S_{N-3}$, and $S_{N-2}$ has been defined as the "context" for the response to $S_{N}$. When context was high, and $S_{N-1}$ was 1 or 2 , and $S_{N}$ was 5 or 6 , the average response to $S_{N}$ was 4.4 , an underestimation of 1.1 categories. When context was low, and $S_{\mathrm{N}-1}$ was 9 or 10 , and $S_{\mathrm{N}}$ was 5 or 6 , the average response to $S_{N}$ was 6.6 , an overestimation of 1.1 categories. These systematic errors, found on trials selected on the basis of the stimuli on the four trials preceding them, can be compared with the plus and minus .8 average error to stimulus 5 or 6 following sequences defined only by $\mathrm{S}_{\mathrm{N}-1}$ (see Fig. 1).

Stimulus sequences can also be selected to yield different levels of accuracy on trials which follow them. For example, the overall average variance of responses to stimulus 10 was .70 and its average per cent correct was $62 \%$. However, when context was high, and $\mathrm{S}_{\mathrm{N}-1}$ was 1 or 2 , the average variance of responses to stimulus 10 was increased to 2.61 and its average per cent correct was decreased to $43 \%$. When context was low, and $S_{N-1}$ was 9 or 10 , the average variance of responses to stimulus 10 was decreased to .07 and its average per cent correct was increased to $91 \%$. These results indicate that the level of performance in an absolute judgment task is critically dependent on the particular schedule of stimuli used. One ought to be able, with the same set of stimuli, to obtain widely different measures of discriminability performance depending on the sequences of stimulus presentations. Since performance in an absolute judgment task is systematically dependent on the preceding sequence of stimuli, the usual consideration of the overall level of performance, for example in terms of information transmission or per cent correct, may not appropriately reflect discriminative capacity.

\section{Discussion}

In an absolute judgment task with feedback provided, it is proposed that Ss use the remembered magnitude of the stimulus on the preceding trial, and the numeric value of the feedback for that stimulus, as a standard for a comparative judgment of the presented stimulus. If this assumption is correct, it would be expected that, as the difference in category steps between the standard and the comparison stimulus increased, the variability of responses would also increase. Variability of responses in the present data increased with increasing difference between the stimulus on trial $\mathrm{N}$ and the stimulus on trial $\mathrm{N}-1$ (Fig. 2). This result provides support for the assumption that a comparative judgment process is involved.

If $\mathrm{Ss}$ perform in an absolute judgment task with feedback as they perform in a comparative judgment task, systematic errors in responses can be attributed to systematic errors in: (1) the memory of the magnitude of the standard $\left(\mathrm{S}_{\mathrm{N}-1}\right)$; (2) the perception of the magnitude of the comparison stimulus $\left(\mathrm{S}_{\mathrm{N}}\right)$; (3) the judgment of the difference, in category steps, between the magnitudes of the presented stimulus and the memory of the standard; or (4) the memory of the numeric feedback assigned to the standard. For the model described here, systematic response errors are attributed solely to a faulty memory of the magnitude of the standard. The perception of the magnitude of the presented 
stimulus and the remembered value of the feedback for the standard are assumed to be veridical. No systematic errors are assumed in the judgment of the difference between the memory of the standard and the magnitude of the comparison stimulus.

Two assumptions are made about the memory of the magnitude of the standard $\left(S_{N-1}\right)$. First, it is assumed that the representation of a simple sensory event in memory is contaminated by the residual traces of prior stimuli in the series. Subjects cannot, or do not, remember the magnitude of a recent stimulus isolated from previous stimuli in the task. For an absolute judgment task with feedback, the memory of the magnitude of the stimulus one trial back (the standard) is affected by prior stimulus context. The context is represented as a distribution of residual traces in memory, from stimuli two and more trials back, into which the magnitude of the stimulus one trial back becomes embedded. The remembered magnitude of the stimulus on the preceding trial, contaminated by the residual traces from previous stimuli in the series, is shifted toward the mean of the distribution of residual traces. The result of this process is the well-known centraltendency effect in which the remembered value of a standard is shifted toward the average of the stimulus series (Needham, 1935).

The second assumption is that memory traces of prior stimuli decay. This assumption has support from several studies, e.g., Brown (1958) and Posner (1964). Anderson (1967) has shown that the contribution of stimuli to a judgment of the mean of the series is an increasing function of recency. If memory traces decay with time or trials, the mean of the distribution of contaminating traces from previous stimuli is a function weighted for recency. Thus, the remembered value of the stimulus one trial back is affected more by the stimulus two trials back than by the stimulus three trials back.

The assumptions for the task reported here, then, are: (A) For an absolute judgment task with feedback, the stimulus one trial back serves as a standard for comparative judgment; (B) The memory for this standard is contaminated by, and thus is displaced toward, traces of prior stimuli; (C) The extent of the contamination by a prior stimulus is an increasing function of recency; and (D) The memory of the numeric feedback one trial back is generally veridical.

The diagram in Fig. 5 illustrates the processes assumed. The $\mathrm{S}$ determines the difference, in category steps, between the magnitude of the presented stimulus $\left(S_{N}\right)$ and his memory of the magnitude of the preceding stimulus (the standard), $R\left(S_{N-1}\right)$. This step-difference, minus two in the example, is then applied to the known correct response (feedback) for the preceding stimulus $\left(\mathrm{S}_{\mathrm{N}-1}\right)$, yielding a response to the presented stimulus. If this difference

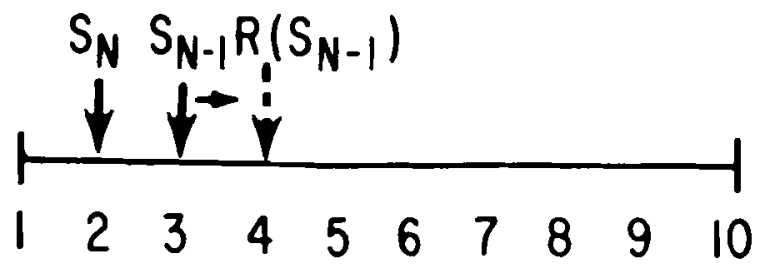

Fig. 5. The memory of the value of the stimulus on trial $\mathrm{N}-1$ is diagrammed as shifted towards the mean of the several stimuli which occurred before it. Generally, this mean will be towards the mean of the stimulus distribution. See text for explanation.

were close to zero, the response to the presented stimulus would be the feedback (true value) of the preceding stimulus. In the diagrammed case, this difference is two steps, and $\mathrm{S}_{\mathrm{N}}$ will be called "one" more often than its true value, "two."

Responses will be wrong, on the average, by the extent to which the remembered magnitude of the preceding stimulus $R\left(\mathrm{~S}_{\mathrm{N}-1}\right)$ differs from its true magnitude $\left(\mathrm{S}_{\mathrm{N}-1}\right)$. Whenever the context, the stimuli immediately preceding $\mathrm{S}_{\mathrm{N}-1}$, is higher than that stimulus, the memory of $\mathrm{S}_{\mathrm{N}-1}$ will be higher than the true value of $\mathrm{S}_{\mathrm{N}-1}$. When the preceding context is lower than the value of $S_{\mathrm{N}-1}$, the memory of that stimulus will be lowered. As diagrammed in Fig. 5, whenever the memory $R\left(S_{N-1}\right)$ is higher than $S_{N-1}$, the current stimulus $\left(\mathrm{S}_{\mathrm{N}}\right)$ is underestimated. On the average, the remembered value of a low stimulus will be higher than its true value and the remembered value of a high stimulus will be lower than its true value. This is the case simply because a number drawn at random from a set of numbers varying from 1 to 10 has an expected value of less than a high number and more than a low number. Stimuli, therefore, tend to be estimated-on the average-as low following a low stimulus and high following a high stimulus. This results in assimilation between the response and the preceding stimulus.

The remembered value of a low stimulus is shifted toward the mean of the distribution of residual traces from prior stimuli (stimuli two or more trials back). The higher the mean of the distribution, the greater will be the upward shift. The greater the upward shift of the memory for the standard, the greater will be the underestimation of the presented stimulus. Therefore, the higher the mean of the distribution of prior stimuli, the lower the average response to the presented stimulus. Conversely, the lower the mean of the distribution of prior stimuli, the higher the average response to the presented stimulus. Thus, contrast should result between the response and the stimuli two or more trials back. Further, the magnitude of this effect should be an increasing function of recency according to the assumption of a decay of memory with time or trials. The results reported in Figs. 3 and 4 confirm these expectations. 
By assuming the processes of contamination and decay in memory, the model provides an explanation of assimilation between the present response and the stimulus one trial back and yields the expectation of a reversal of this effect (contrast) for stimuli two or more trials back. Observed changes in the means and variances of response distributions were consistent with this model. From this point of view, assimilation and contrast effects a re not independent judgmental processes, but are the response consequences of specified memorial processes.

\section{References}

Anderson, N. H. Application of a welghted average model to a psychophysical averaging task. Psychon. Sci., 1967, 8, 227-228.

Barry, H., III. Effects of prolonged deliberation on psychophysical judgment. Amer. J. Psychol., 1964, 77, 270-275

Brown, J. Some tests of the decay theory of immediate memory.
Quart. J. exp. Psychul., 1958, 10, 12-21.

Gamer, W. An informational analysis of ausolute judgments of louduss. J. cxp. Psychol., 1953, 46, 373-380.

Helson, II. Adaptation livel theory. In S. Koch (Ed.), Psycholoon. A study of a sciencé Vol. 1. New York: McGraw-Hill, 1959

Needham, J. G. The efle $t$ ff the time interval upon the time-error at different intensivo levels. J. exp. Psychol, 1935, 18, 530-543.

Parducci, A., \& Marshall, L. M. Assimilation vs. contrast in the anchoring of perceptual judgments of weight. J. cxp. Psychol. $1962,63,426-437$.

Parducci, A., Sandusky, A. Distribution and sequence effects in judgment. $J$. exp. Psychol., 1965, 69, 450-459.

Pollack, 1. Order effects in the category scaling of grays. Psychon. Sci., 1964, 1, 69-70.

Posner, M. 1. Rate of presentation and order of recall in immediate memory. Brit. J. Psychol., 1964, 55, 303-306.

\section{Noie}

1. This research was supported in part by a NASA traineeship grant to the first author and by Research Grant GB 4916 from the National Science Foundation.

(Accepted jor publication February 6, 1968.) 